\title{
Research on target location technology of campus video surveillance system based on WBAN and Internet of things \\ GAO Jie ${ }^{1}, \mathrm{HUANG} \mathrm{Yi}^{2}$ \\ \{oijsfdiosd984@163.com¹,jahg245@163.com²
}

(1. Modern Economics \& Management College of JXUFE,Gongqingcheng 332020,China; 2.NanChang Business College of jxau,Gongqingcheng 332020,China)

\begin{abstract}
In the traditional monitoring process, due to too much subjectivity of people, and the human brain's attention to things is accurate, but the sustainability and diversity are poor. When the monitor observes several surveillance cameras at the same time, the accuracy will be greatly reduced with the increase of time.Based on this, a research on target location technology of campus video surveillance system based on WBAN and Internet of things is proposed.Firstly, a three-dimensional geographical scene was constructed to detect the moving target, then the feature of the target was extracted and the image was preprocessed, and the target positioning was realized through the generation of panorama and perspective.Experimental results show that the proposed method has high positioning accuracy.
\end{abstract}

Keywords: WBAN; The Internet of things;Campus video surveillance;target.

\section{Introduction}

With the development of industrial society, information security and social security have become the hottest topics.As an important information technology, video surveillance has been paid more and more attention by the society in the field of security.However, with the popularization and enlargement of the monitoring system, the traditional human-dependent monitoring cannot meet the needs of today's society more and more, and its limitations become more and more prominent [1].In the traditional monitoring process, due to too much subjectivity of people, and the human brain's attention to things is accurate, but the sustainability and diversity are poor. When the monitor observes several surveillance cameras at the same time, the accuracy will be greatly reduced with the increase of time.On the one hand, the monitor can't observe many things at the same time. On the other hand, the monitor can't pay attention to monotonous things for a long time.Finally, human physiology needs rest and diet, which also affects the reliability and stability of the monitoring system. All of these factors have led to the problems of the traditional human-dependent monitoring system, including high failure rate, poor reliability, and slow corresponding speed.At the same time, due to the huge and complex monitoring system, if all rely on human monitoring, the human cost is quite expensive, it is difficult to maintain such a large investment in the actual monitoring system.

WBAN, Wireless Boay Area Network (WBAN, Wireless Boay Area Network) is a communication Network that centers on the human body and aims at collecting various physiological parameters of the human body.Through WBAN, people can communicate and synchronize data with their personal electronic devices (such as pdas and mobile phones).WBAN can communicate with other data communication networks (such as other people's WBAN, wireless/wired access network, mobile communication network, etc.) as part of the entire communication network, and communicate with any terminal on the network (such as PC, mobile phone, telephone, media player, digital camera, game console, etc.).With 
the increasing complexity of monitoring scenes, the traditional video surveillance analysis based on two-dimensional images can no longer meet people's needs, and more information needs to be obtained from scenes to add to the analysis factors [2].However, in today's monitoring, depth information becomes particularly important. Therefore, the positioning technology of obtaining the three-dimensional space point coordinates corresponding to moving objects from $2 \mathrm{~d}$ images based on the camera model has become a hot issue in intelligent monitoring technology.

\section{Target location technology of campus video monitoring system based on WBAN and Internet of things \\ 2.1 Construction of $3 \mathrm{~d}$ geographical scenes}

The target location technology of campus video surveillance system is realized on the basis of constructing realistic three-dimensional geographical scene, which also provides detection environment parameters for the combination of WBAN and Internet of things. The purpose of this study is to reconstruct the $3 \mathrm{~d}$ geographical scene in order to simulate the target on the surface of the video surveillance area and provide the virtual object for the virtual camera imaging.In the case that the monitoring area is bare land, high-precision DEM data can be directly used, or $3 \mathrm{~d}$ laser scanner can be used to scan the area to form point cloud data, and then gis can be used to form the earth surface model (DSM) of regular grid [3].3d scene rendering can be done using osgEarth, OSG's third-party terrain rendering tool.The algorithm idea of osgEarth is to use quadtree data structure to divide terrain data and texture data into layers and blocks, build pyramids of different resolution of digital terrain and texture images, and establish terrain block nodes of different LOD layers [4].This rendering method is beneficial to improve the efficiency of scene modeling.

\subsection{Moving target detection}

The moving target is moving and changing, so it needs to be detected to provide a basis for its precise positioning. This step mainly USES the displacement sensor in the wireless sensor node of WBAN to detect the moving target by comparing the input image with the background reference image.Sensor is a kind of high performance sensor used to measure three-dimensional motion attitude. This type of sensor is mainly a measurement System based on Micro Electro Mechanical System (MEMS) technology.Generally, the MEMS system consists of triaxial gyroscope, triaxial accelerometer, triaxial electronic compass, triaxial magnetic sensor, triaxial angular velocity sensor, triaxial acceleration sensor and other motion sensors.

Set the background reference image at time $\mathrm{n}$ as $f_{\mathrm{ln}}(x, y, n)$ and the current frame as $f_{e}(x, y, n)$, then the background difference image is:

$$
f_{d}(x, y, n)=\left\{f_{0}(x, y, n)-f_{s}(x, y, n)\right\}
$$

Formula (1) shows that the background difference value of each pixel is $f_{d}(x, y, n)$, so we can get the change detection mask as follows:

$$
c(x, y, n)\left\{\begin{array}{l}
1, \text { iff }_{e}(x, y, n)>T \\
0, \text { other }
\end{array}\right.
$$

The judgment basis of moving target detection is as follows: if the difference value is greater than $T$, then $(x, y)$ points belong to the moving target pixel, and vice versa.The $T$ here is the threshold.The change detection mask set at the time of update interval $n$ 
and $n-1$ is $c(x, y, n)$ and $c(x, y, n-1)$ respectively, then the motion template $M(x, y, n)$ at the time of $\mathrm{n}$ is obtained from the images of the two adjacent update intervals, as follows:

$$
M(x, y, n)=C(x, y, n-1) / C(x, y, n)
$$

$M(x, y, n)$ is a target factor that determines which pixels in the current frame are used to update the current background. The background obtained according to formula (4) is called the instantaneous background, and then according to formula (5), the weighted average of the instantaneous background and the current background is used to obtain the current new background.

$$
\begin{gathered}
f_{b}(x, y, n)=\left\{\begin{array}{l}
f_{e}(x, y, n), i f M(x, y, n)=0 \\
f_{d}(x, y, n-1), i f M(x, y, n)=1
\end{array}\right. \\
f_{e}(x, y, n)=\partial_{b}(x, y, n)+(1-\partial) f_{b}(x, y, n-1)
\end{gathered}
$$

Where ${ }^{\partial}$ is the update factor, ${ }^{\partial}$ affects the background update speed, which is generally selected by the experiment.After several experiments, ${ }^{\partial}$ is selected as 0.1 .

Hall Monitor sequence was selected for the experimental video stream. The resolution of the image frame was $352 \times 288$, and the image was frame 40 .The experimental platform is celeron $1.7 \mathrm{~g}, 256 \mathrm{mb}$ of memory, compiled with $\mathrm{VC}++$, processing speed of about 15 frames/second.The generation process of the new background in this paper is set as (a), (b), (c), (d), (e), (f), and (a) is the current frame $f_{c}(x, y, n)$;(b) is a motion target template;(c) background $f_{b}(x, y, n-1)$ of the previous frame;(d) and (e) are the instantaneous background corresponding to the image when $M(x, y, n)$ is 0 and 1 respectively;(f) new background image for final production.

\subsection{Target feature extraction}

When object detection is conducted in the video, the purpose is to identify and detect the part of the region (target region) that you are interested in from the complex and changeable background environment, and then calculate the general trajectory of the target to achieve accurate target location.In general, three criteria of real-time, accuracy and robustness are used to judge the target tracking system [5].Real-time is in the detection of the target, the system is expected to be real-time, continuous detection;Accuracy is in the detection of the target, hope to be able to correctly detect the target as accurate as possible;Robustness means that when the external environment is constantly changing, the detection system is expected to be robust enough to ensure the effectiveness and feasibility of detection.Usually, when detecting and tracking the target, a feature of the target is selected to match with the video image sequence.The selection of features is related to the accuracy of subsequent positioning.The commonly selected target features include color features, edge features, texture features and shape features.

Color features.In the description of color space, RGB color space and HSV color space are usually used more.The RGB color space is a color space composed of red (R), green (B), and blue (G).HSV color space is a color space composed of chroma, saturation and brightness.In the description of color features, there are many ways to use color histogram, which specifies the approximate distribution information of color, and the effect is 
better.However, only the quantitative distribution information of the color is specified, and the position information of the color body itself is not specified.If similar color distribution information appears near the tracking target, it will lead to tracking error.

Marginal features.Edge feature is mainly the edge distribution information of the image.The edge information characteristics described in this way are better tracked on general targets.If the edge characteristics of the target to be tracked are not so obvious, the tracking effect based on this method is not ideal.

Texture features.Texture features represent a relationship between the pixels of the target region in the image.It is only a statistical feature on the surface of the target, with strong regularity.In this way, the influence of noise can be effectively avoided.

Shape characteristics. Shape feature is to extract some shape information of the known edge region.The shape information includes the shape information of the area and the shape information of the target contour.In this way, the shape information of the whole object can be obtained.

Directional gradient characteristics.The feature information of the direction gradient is obtained by calculating and constructing the histogram of the direction gradient in an area of the image.By dividing the image into several smaller parts, the histogram of the direction gradient of each small region is calculated. The histogram of the direction gradient of each small region can uniquely describe the information of this region.

\subsection{Image preprocessing}

By high speed CCD camera filmed for the rail fastener continuous video, consecutive video frames is actually made up of many images of (general collection of video is 25 pour/seconds, namely a second 25 images), WBAN and Internet of things through the lowpower embedded ARM processor in the system, you can get the three-dimensional human body stance, Angle and azimuth data.In addition, quaternion algorithm and data fusion technology can also be used to obtain special data, and the output data are usually collected in the form of quaternion and euler Angle.Need to be aware of is the use of WBAN continuously and comprehensive data acquisition and the Internet of things will produce a large amount of data, how to filter integration of useful data, eliminating redundant information, which is a key research question, the key is the need of image preprocessing, which must be poured video into images, including smoothing denoising and extract the image edge.

Main purpose of image smoothing can reduce the noise, the noise influence on image signal amplitude and phase is very complex, some noise and image signal are relevant, some have no correlation, also may have a correlation between the noise itself, so if you want to reduce the noise in the image, must according to specific situation adopt corresponding measures, smooth denoising is a kind of can suppress the image noise reduction and image processing technology, from the perspective of the image quality is the main purpose of image enhancement is to improve the image of intelligible degree, can make the enhanced image is more suitable for the human eye observation or is more suitable for machine vision recognition system, When the camera located at the bottom of the monitoring system collects the video of the target coupler, the image quality will inevitably be reduced due to various interferences such as the external environment. In this paper, WBAN enhancement method is used to enhance the image [6], which will obtain good image quality.

The edge of the image is the most basic feature of the bottom layer of the image. The edge information of the object is expressed in the form of the discontinuous local characteristics of the image, such as the change of image gray level, the change of image color and so on.Edge detection can greatly reduce the amount of data, and remove the information that we think is irrelevant and only keep the important attributes of the image structure.In this 
paper, edge extraction is divided into two processes: training and testing [7]. In this paper, the rail processing images have rich texture features and the grey value change [8], so this article selected two characteristics to process the image brightness and texture, using machine learning methods, the sleeper edge character and the edge feature extract and obtained the parameter values, we need training process is as follows:

First, 50 images were selected as the training sample set.

Second, the edge segment and non-edge segment of the training sample set are marked. This paper is marked red, so the pixel point can be judged to be on the edge or non-edge point according to the $\mathrm{R}$ channel value of the image.

Third, randomly get a certain number of each image pixel, three over ten of them are case also is the point of the edge of the sleeper, seven over ten take negative cases is also edge points, and then calculate the histogram of each operator eigenvalue, because of the brightness, texture feature value has eight directions, and we focus on close to the brightness of the horizontal direction or texture characteristic value, so only select 0 , PI / 8, $7 *$ PI / 8 three directions, computation formula is as follows (6):

$$
x^{2}(g, h)=\frac{1}{2} \sum_{i}^{M}\left(\frac{g_{i}-h_{i}}{g_{i}+h_{i}}\right)^{2}
$$

$\mathrm{X}$ stands for pixel point, $\mathrm{g}$ for brightness, and $\mathrm{h}$ for image height.

Finally, the calculated eigenvalues are sent to the classifier for training. The function selected in this paper is:

$$
Y=\frac{1}{\left(1+\exp \left(-x^{2}\right)\right)}
$$

Fifth, the trained parameter beta value is calculated.

Secondly, the process of testing. The parameters obtained from training are put back into the original program. Through comparison and research, this paper selects the characteristic values with a calculation radius of 0.025 and a calculation direction of three directions, namely the final test effect diagram, it can be found that the edge of the sleeper can be clearly detected.The edge detected is a discontinuous edge pixel, and the eight connected regions of the pixel are connected to the corresponding length of the line segment, so as to obtain the required rail edge segment and sleeper edge segment.

\subsection{Generation of panorama and perspective}

Generally, the omni-directional image obtained by the monitoring system is an omnidirectional image. Since the omni-directional image is a compressed circular panoramic image, it is necessary to expand the image in order to make it consistent with human observation and understanding habits. At the same time, it is also convenient to use the computer for further analysis and processing. When the panorama is expanded, the $360^{\circ}$ ring information can be obtained. When the perspective is expanded, it is more in line with the perspective observed by the human eye, facilitating the processing of details. Meanwhile, relative to the panorama, some spatial distortion phenomena in the generated image are reduced [9]. 
The development process of the omni-directional image is the inverse process of the omni-directional image generation process. In the process of the panoramic image, the omnidirectional image on the CCD is expanded to a cylinder in space. During the process of perspective expansion, some points in the omni-directional image are restored to a specific point in space. When the panorama is expanded, all points in the omni-directional image are mapped to the cylinder with radius $\mathrm{L}$, elevation $\phi 1$, and depression $\phi 2$. Any point $P(X, Y, Z)$ on the cylinder satisfies the following conditions:

$$
x^{2}+y^{2}=L^{2}
$$

The cylinder is crosscut by $\mathrm{y}=0$ and $\mathrm{x}=\mathrm{L}$, and the cylinder is drawn into a plane from the xy plane in the order of $1,2,3$ and 4 quadrants. The points mapped to the plane are a panorama generated by the omni-directional image. The coordinate origin of the panorama is the point where the $\mathrm{x}$ axis intersects the cylinder in the positive direction.In the spatial coordinate system, the $\mathrm{z}$-axis coordinate value is the $\mathrm{Y}$-axis coordinate value of the panorama. Here, the intersection line of the original xy plane and cylinder is taken as the positive direction of the x-coordinate of the panorama along the stretching direction, and the corresponding relationship between the following coordinates of the panorama (x1, y1) and the omnibearing image $(\mathrm{x}, \mathrm{y})$ is obtained:

$$
\begin{gathered}
x=\frac{\cos \left(x_{1} / 2 L\right)}{2 a c\left(a^{2}+c^{2}\right)} \\
x=\frac{\sin \left(x_{1} / 2 L\right)}{2 a c\left(a^{2}+c^{2}\right)}
\end{gathered}
$$

In the viewpoint of the focus of the hyperboloid namely space coordinate origin generated perspective, for mapping plane is $G$, in the center of the plane as the origin of coordinates, a bit in the plane of the G (gx, gy) and space coordinates, the origin of the lines in the $\mathrm{x}, \mathrm{y}$ plane projection with the $\mathrm{x}$ axis is the direction of the crossing Angle for Ox, and the line in the plane of projection and axis is the direction of Oy, get a $\mathrm{G}$ (gx, gy) corresponding relation with the space coordinates:

$$
\begin{gathered}
x=\left(L \cos O_{y}+g_{y} \sin O_{y}\right) \\
y=\left(L \cos O_{y}+g_{y} \sin O_{y}\right) \sin O_{x} \\
z=L \sin O_{y}-g_{y} \cos O_{y}
\end{gathered}
$$

Before generating the panorama and perspective, the first thing to determine is the value of the endpoint coordinates in the direction of the height of the required generated image, that is, to determine the $\mathrm{z}$ value of the two endpoints on the $\mathrm{Y}$-axis of the panorama in the spatial coordinate system.Among them, one method is to calculate the spatial coordinates of the upper and lower endpoints by expanding the length of the axis after calculating elevation $\phi 1$ and depression $\phi 2$ through a and b. This method is simple and does not require prior knowledge of the inner and outer diameters of the omni-directional image.Another way is to take the $\mathrm{xz}$ 
plane in space, and get the points in space for $r$ and $r$.The transformation formula is obtained through mathematical analysis as follows:

$$
y=\frac{2 a c \sqrt{f^{2}+x^{2}}-f L\left(a^{2}+c^{2}\right)}{b_{2} x}
$$

The advantage of this method is that when the inner and outer diameters are manually delineated on the omni-directional image, the generating range of panorama and perspective can be changed, and the data deviation caused by the fixing device on the omni-directional lens shell to the first method can be avoided [10].After the coordinate values of the upper and lower vertices of the height direction of the generated graph are determined by the above method, the respective $\mathrm{Y}$-axis coordinate values of the top and bottom of the panorama are the coordinate values of the upper and lower vertices.After determining the top and bottom coordinates of the panorama, the value range of the upper and lower endpoints in the perspective can be obtained through trigonometric analysis. The process of generating a panorama and perspective is the process of traversing each point in the generated empty image and starting to find the corresponding point on the omnibearing map.

In the process of panorama generation, it can be found from the corresponding calculation formula that, except for the calculation part of trigonometric function, which is related to the width value $\mathrm{x}$ of the image, most of the calculation is only related to the height value y.In this way, when traversing every point in the panorama, the points in the height direction should be taken as an external loop, so that only the data related to the X-axis should be calculated in traversing every row, thus greatly reducing the calculation amount of the algorithm.In the process of omni-directional image development, the selection of $\mathrm{L}$ is a key problem. The selected value of $\mathrm{L}$ is directly related to the computational amount of the computer, the quality of the generated image, and the pixel loss and repetition of the image.Of course, the value of $\mathrm{L}$ can be fixed according to the requirement of panoramic display width $\mathrm{W}$ in the project. The advantage of this method is that when the image resolution is large, the calculation amount can be minimized according to the need of display. At this point:

$$
L=\frac{W}{2 \pi}
$$

In general, the generation height of the image can be generated $\mathrm{h}=\mathrm{R}-\mathrm{r}$, where:

$$
L=\frac{h}{(\phi 1+\phi 2)}
$$

At this point, the value of $\mathrm{L}$ varies according to the height of image generation. The advantage is that when the resolution of the original image is different, the quality and speed 
of image generation can be effectively guaranteed.In the practical engineering application, we can combine the two and judge and choose according to the actual situation.If the resolution of the original image is $L=\frac{h}{(\phi 1+\phi 2)}$ within a certain range, and the resolution of the original image is large enough to make the panorama take a long time to generate, then $\mathrm{L}$ is considered to be fixed to save the expansion time.

The expansion of the panorama maps the imaging points in space to a cylinder with the zaxis as the axis.When an object in space is not parallel to the z-axis, due to its mapping relationship, it will cause distortion in the z-axis direction after the object is projected. The larger the Angle of intersection with the z-axis, the greater the distortion will be.Figure 2 is an example of image distortion. The line segment $\mathrm{L} 1=\mathrm{L} 2$ in the space is not the same length when expanded into a panorama, resulting in distortion in the panorama.Therefore, in order to obtain an accurate positioning in the final positioning link, it is necessary to generate the standard panorama as far as possible to reduce image distortion.

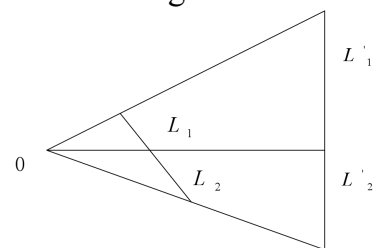

\subsection{Target positioning}

Fig. 1. Distortion during omni-directional panoramic projection

The final implementation process of the target positioning of campus video monitoring system based on WBAN and Internet of things, namely, the final target positioning process is as follows:

The first step is to scan the image line by line and search for the pixel with a gray value of 255 in the image.Set to get the first gray value is 255 pixels in the image $\left(x_{0}, y_{0}\right)$, have $\left(x_{0}, y_{0}\right)$ as the seed pixels onto the stack, and give it marked as a motion object Movingobj0 (also is a prospect target), and the object pixel count set to 1, the grey value of pixels in the current frame $f_{0}\left(x_{0}, y_{0}\right)$ as seed pixel gray value, $M\left(x_{0}, y_{0}, n\right)$ assigned a zero value.

The second step is to take out the seed pixel $f_{0}\left(x_{0}, y_{0}\right)$ from the stack. The gray value of the pixel $f_{0}\left(x_{0}, y_{0}\right)$ in the current frame is taken as the gray value of the seed pixel. Taking it as the center, the neighborhood pixel is checked.If the domain pixel $\left(x_{1}, y_{1}\right)$ satisfies $M\left(x_{0}, y_{0}, n\right)=1$, the pixel is considered to be part of Movingob and pushed onto the stack.Otherwise stop the area from growing further.In order to accelerate the search speed, this paper USES the $5 \mathrm{X} 5$ neighborhood window to search.That is:

$$
M\left(x_{1}+k, y_{1}+m, n\right)=\frac{1}{f_{n}\left(x_{1}, y_{1}\right)}
$$


After scanning the 5 X5 domain pixel $\left(x_{0}, y_{0}\right)$ of the pixel, go back to the beginning of this step until there are no seed pixels in the stack.

Third, determine the minimum box containing the moving object Movingobj0.Its target is within range of region $\left(x_{0}, y_{0}\right)\left\{(x, y) \mid x_{\min } \leq x \leq x_{\max }\right\}$.

Fourth, repeat the above steps until the gray value in the image is zero.

Fifth, according to the number of pixels count of each moving object, remove the moving objects whose count is less than the range, because these are often false targets formed by noise or unprocessed shadows. The following description of the target motion can usually be achieved by describing the trajectory of the target center of mass.

\section{Test experiment}

In this paper, a target positioning technology of campus video surveillance system based on WBAN and Internet of things is designed. In order to verify the practical application effect of this method, a comparative experiment is designed with the common monitoring system target positioning method.Through comparison, the experimental hypothesis is verified.

\subsection{Experimental contents}

In the experiment, a school playground was used as the experimental site. Four cameras were installed in the site to shoot a group of video simultaneously.Experiment site pedestrians in 89 , as shown in figure 2 and figure 3 is one of the cameras interval of $10 \mathrm{~s}$ acquisition of image information, this section is the red box in figure 2 and figure 3 identification of target localization experiment, the blue box identification of target tracking experiment, set a goal according to the given trajectory motion, one goal some shade.

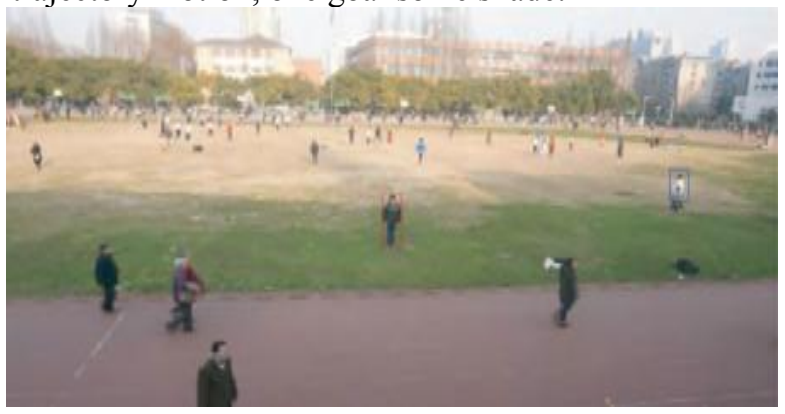

Fig. 2. Target position 1 


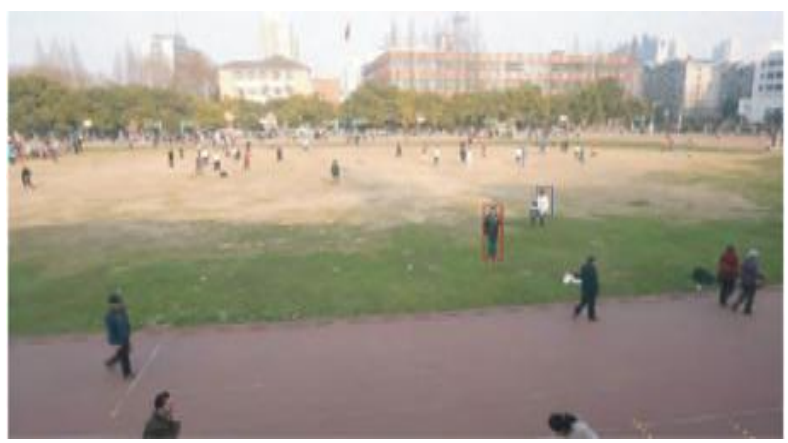

Fig. 3. Target position 2

\subsection{Multi-angle target positioning test and analysis in video monitoring}

For the target identified by the red box in FIG. 2 and FIG. 3, the proposed method and the traditional method are respectively adopted to locate the target from different perspectives. The positioning results of target position 1 and 2 by the two methods are shown in table 1 and table 2 .

Table 1. Location results of two methods of target location 1

\begin{tabular}{|c|c|c|c|}
\hline Angle of view & $\begin{array}{c}\text { Actual } \\
\text { coordinates } / \mathrm{m}\end{array}$ & $\begin{array}{l}\text { The method of } \\
\text { this paper }\end{array}$ & $\begin{array}{l}\text { conventional } \\
\text { method }\end{array}$ \\
\hline 1 & $(366.27,394.45)$ & $(366.27,394.46)$ & $(369.67,396.59)$ \\
\hline 2 & $(366.27,394.45)$ & $(366.26,394.41)$ & $(370.21,398.32)$ \\
\hline 3 & $(366.27,394.45)$ & $(366.27,394.44)$ & $(371.25,399.12)$ \\
\hline 4 & $(366.27,394.45)$ & $(366.28,394.42)$ & $(369.58,397.89)$ \\
\hline \multicolumn{4}{|c|}{ Table 2. Location results of two positioning methods } \\
\hline Angle of view & $\begin{array}{c}\text { Actual } \\
\text { coordinates/m }\end{array}$ & $\begin{array}{l}\text { The method of } \\
\text { this paper }\end{array}$ & $\begin{array}{c}\text { conventional } \\
\text { method }\end{array}$ \\
\hline 1 & $(373.19,394.75)$ & $(373.22,394.72)$ & $(375.86,397.21)$ \\
\hline 2 & $(373.19,394.75)$ & $(373.18,394.72)$ & $(377.68,399.83)$ \\
\hline 3 & $(373.19,394.75)$ & $(373.25,394.78)$ & $(370.12,391.17)$ \\
\hline 4 & $(373.19,394.75)$ & $(373.19,394.28)$ & $(369.26,396.55)$ \\
\hline
\end{tabular}

It can be seen from the analysis of table 1 and table 2 that, compared with the traditional method, the positioning coordinates obtained by the method in this paper are closer to the actual coordinates for targets from different perspectives.

In order to verify the positioning accuracy of the proposed method more directly, the positioning errors of the proposed method and the traditional method are compared on the basis of the above experiments. The calculation formula of positioning error is as follows:

$$
d=\sqrt{\left(x_{t}-x_{1 t}\right)+\left(y_{t}-y_{1 t}\right)}
$$

In equation (18), 1 is used to describe the real position coordinates of the target; 2 is used to describe the target position coordinates obtained by different methods. On the basis of the above experiments, the positioning errors of the method in this paper and the spring method 
are calculated through equation (18), and the comparison results are described in table 3 and table 4.

Table 3. The comparison results of positioning errors between two positioning methods of target

\begin{tabular}{ccc}
\multicolumn{3}{c}{ position 1 } \\
\hline angle of view & $\begin{array}{c}\text { The method of } \\
\text { this paper/m }\end{array}$ & $\begin{array}{c}\text { conventional } \\
\text { method/m }\end{array}$ \\
\hline 1 & 0.06 & 0.13 \\
2 & 0.09 & 0.18 \\
3 & 0.07 & 0.20 \\
4 & 0.11 & 0.16
\end{tabular}

Table 4. The results of positioning error comparison of two positioning methods of target position 2

\begin{tabular}{ccc}
\hline angle of view & $\begin{array}{c}\text { The method of } \\
\text { this paper/m }\end{array}$ & $\begin{array}{c}\text { conventional } \\
\text { method } / \mathrm{m}\end{array}$ \\
\hline 1 & 0.03 & 0.15 \\
2 & 0.06 & 0.21 \\
3 & 0.05 & 0.17 \\
4 & 0.07 & 0.25 \\
\hline
\end{tabular}

It can be seen from table 3 and table 4 that the maximum positioning error of the method in this paper is 0.11 , lower than the minimum positioning error of the traditional method, which indicates that the positioning accuracy of the method in this paper is higher than that of the traditional method and the positioning performance is excellent.

\section{Conclusion}

This paper analyzes the target positioning technology of campus video surveillance system based on WBAN and Internet of things, and locates the target through the method of WBAN and Internet of things. The local function is obtained from the video data in different angles.According to the generation of panorama and perspective to achieve information fusion, to achieve the goal of positioning.Experimental results show that the proposed method has high positioning accuracy.It is hoped that the research in this paper can provide theoretical basis for the target positioning method of campus video surveillance system based on WBAN and Internet of things.

Headings. Please follow the formatting instructions for headings given in Table 1.

Tables. All included tables must be referred to in the main text and the table title and caption are to be positioned above the table.The captions need to be written in Times New Roman, 9pt.

\section{Fund project}

Science and technology project of Jiangxi Provincial Department of education,

Project No.: GJJ171481, Project Name: Research on Key Technologies of campus video monitoring system based on Internet of things, Project type: Youth Project

\section{References}

[1]Wang Yan Yu. Design of Intelligent Campus Security Monitoring Internet of things system.Modern Electronic Technology, 42 (6): 51-51 (2019)

[2]Ji Wenwen, Ren Yongqiong. Research on Intelligent Management system of computer Laboratory based on Internet of things. Computer products and Circulation, 12(06): 146-147 (2019)

[3]Ren Bingjia. Application of Internet of things technology in safety management of cadre college. Information and computer (theoretical Edition), 2(11): 184-185 (2019) 
[4]Li Yewei, Sun Qunfeng. Research on Smart Park Operations Management System Based on Cloud Computing. ICT,13(2):58-62+69 (2019)

[5]Deng Jiaming, Zhang Zhipeng, Pan Xiting. Research on Intelligent Campus Student behavior Management system based on face recognition. Journal of Anshan Teachers College, 21(2): 50-54 (2019)

[6]Wu Heng, Xiao Qia, Liao Xiaojian. Design of Laboratory Safety Monitoring System Based on IoT Technology. IoT Technology,9(7):24-26 (2019)

[7]Jiang Xiangkui, Ning Yuwen, Fan Yongqing, Shang Zitian, Zhang San. Design of visual management platform for intelligent classroom based on Internet of things. China Educational Informatization,5(17): 42-45 (2019)

[8]Ho Chi, Huang Yanglai, Wang Mengdi. Design of face recognition campus monitoring system based on Internet and STM 32 F 4 single chip machine. Intelligent Robot, 12(05):78-80+85 (2019)

[9]Wang Lu, Zhou Yimin, Yu Zhihao. Design of Intelligent Laboratory Security System Combining Campus Card System and Internet of Things Technology. Electronic Devices,41(04):1077-1081 (2018)

[10]Shen Hui. Research on Campus Monitoring Technology Based on Internet of Things Technology. Heilongjiang Science,9(16):130-131(2018) 